\title{
MATROID REPRESENTATIONS AND FREE ARRANGEMENTS
}

\author{
GÜNTER M. ZIEGLER
}

\begin{abstract}
We show that Terao's Conjecture ("Freeness of the module of logarithmic forms at a hyperplane arrangement is determined by its abstract matroid") holds over fields with at most four elements. However, an example demonstrates that the field characteristic has to be fixed for this.
\end{abstract}

\section{Free ARRANGEMENTS}

The present study continues an investigation of the connection between algebraic and combinatorial structures of hyperplane arrangements $[15,17,23$, 24].

Specifically, the question ("Terao's Conjecture") is studied whether freeness of the module of logarithmic vector fields at an arrangement is determined by the underlying matroid.

Here we apply representation theory for matroids to give positive answers in several important cases (binary matroids, arrangements over $\mathrm{GF}(2), \mathrm{GF}(3)$, and $G F(4)$ ) where the arrangements are essentially projectively unique.

However, it is shown that freeness of the arrangements corresponding to certain matroids does depend on the field characteristic: freeness cannot be recognized from the matroid alone.

For this the technique of supersolvable resolutions is introduced, which allows freeness proofs (and disproofs) that depend on the embedding of the arrangement and thus on the specific representation of the underlying matroid.

This leaves the feeling that Terao's Conjecture may well be wrong, although for several reasons hard to disprove: on the one hand freeness is a "rigidity property", and if a free arrangement is so rigid that it is projectively unique, then it cannot give rise to a counterexample; on the other hand the insight seems to emerge that the only arrangements that, combinatorially and philosophically speaking, have "a right to be free" (in the sense of Terao) are the supersolvable ones.

Our hope is that the present note provides new insight into the combinatorial structure governing the algebraic properties of hyperplane arrangements.

Received by the editors September 16, 1988.

1980 Mathematics Subject Classification (1985 Revision). Primary 05B35, 32C40; Secondary 13H15, 51D25, 51M20. 
Definition and Construction 1.1. An arrangement (over $\mathbf{k}$ ) is a finite set $X=$ $\left\{H_{1}, \ldots, H_{m}\right\}$ of hyperplanes through the origin in a finite dimensional vector space $V=\mathbf{k}^{n}$. We call $n=\operatorname{dim}(V)$ the dimension and $m=|X|$ the order of $X$. The rank of an arrangement is the codimension of the intersection of all its hyperplanes, $r=r(X)=\operatorname{codim}(\bigcap X)$. The arrangement is essential if $r(X)=n$, that is, if $\cap X=\{0\}$. The rank of a set $Y \subset X$ of hyperplanes is $r(Y)=\operatorname{codim}(\bigcap Y)$.

The direct sum of two arrangements $X$ in $V$ and $X^{\prime}$ and $V^{\prime}$ is the arrangement $X \oplus X^{\prime}=\left\{H \oplus V^{\prime}: H \in X\right\} \cup\left\{V \oplus H^{\prime}: H^{\prime} \in X^{\prime}\right\}$ in $V \oplus V^{\prime}$.

For every hyperplane $H \in X$ we select a linear form $l_{H} \in V^{*}$ that defines $H$ (that is, such that $\left.H=\operatorname{ker}\left(l_{H}\right)\right)$.

The abstract matroid $M=M(X)$ defined on $X$ by linear independence on the corresponding vectors in $\left\{l_{H}: H \in X\right\}$ is the (abstract) matroid of $X$. This matroid $M$ is simple: it does not have loops or multiple points. Its lattice of flats is the lattice $L$ of intersections of the hyperplanes in $X$, ordered by reverse inclusion; it is called the intersection lattice of $X$ [22]. Its rank is $r(L)=r(X)$. The matroid rank function of $M$ coincides with the rank function defined above on the corresponding sets of hyperplanes. Thus every hyperplane arrangement canonically determines a represented matroid. Conversely, for every represented simple matroid $R \subseteq V-\{0\}$ there is a canonical arrangement $X_{R}=\left\{H_{v}: v \in R\right\}$ in $V^{*}$ given by $H_{v}=\left\{l \in V^{*}: l(v)=0\right\}$.

With this construction hyperplane arrangements are equivalent to represented simple matroids (represented geometries). This will be an important point of view for much of this paper: we will study arrangements arising from representations of matroids over different fields, their uniqueness and "freeness."

A special case is that of binary arrangements: a matroid is binary if it is representable over $\mathrm{GF}(2)$. An arrangement (over an arbitrary field) is binary if its abstract matroid is binary. It is well known that if $\operatorname{char}(\mathbf{k}) \neq 2$, then every binary matroid representable over $\mathbf{k}$ is in fact regular (unimodular), that is, representable over every field.

The characteristic polynomial of $L$ is denoted by $\chi(t)=t^{r}-m t^{r-1}+\cdots$ and referred to as the characteristic polynomial of $X[19,21]$.

A coatom $h \in L \quad(r(h)=r-1)$ is modular if it intersects every line (flat of rank 2). In this case the characteristic polynomial of $L$ factors as $\chi_{L}(t)=$ $\chi_{h}(t) \cdot\left(t-e_{r}\right)$, where $\chi_{h}(t)$ is the characteristic polynomial of the flat $h$ (of the interval $[\hat{0}, h])$, and $e_{r}$ is the number of atoms of $L$ that do not lie below $h$, that is, $e_{r}=|X|-|h|[11]$.

Recursively, a geometric lattice is defined to be supersolvable [12] if it has rank at most 1 or if it contains a modular hyperplane $h \in L$ such that $[\hat{0}, h]$ is supersolvable. In this case the characteristic polynomial factors over $\mathbf{Z}$ as $\chi(t)=\prod_{i=1}^{r}\left(t-e_{i}\right)$, where $e_{1}=1$ and $\sum_{i=1}^{r} e_{i}=|X|$. The integers $e_{1}, \ldots, e_{r}$ are the exponents of $L$. 
Definition 1.2. An invariant of a class of arrangements is combinatorial if it is determined by the abstract matroids of the arrangements (equivalently, by the intersection lattices).

Let $x_{1}, \ldots, x_{n}$ be a basis of $V^{*}$, such that $S:=\mathbf{k}\left[x_{1}, \ldots, x_{n}\right]$ is the ring of polynomial functions on $V$. We call $Q:=\prod_{H \in X} l_{H} \in S$ a defining equation of $X$ (considering $X$ as a reduced hypersurface in $V$ ). $Q$ is a homogeneous polynomial of degree $m$. Let $\Omega_{\text {alg }}^{1}(V)=S \otimes \Lambda^{1}(V)$ be the $S$-module of algebraic differential 1 -forms on $V$, with its natural grading by polynomial degree, decomposing as

$$
\Omega_{\mathrm{alg}}^{1}(V)=\bigoplus_{k \geq 0} \Omega_{\mathrm{alg}}^{1, k}(V) .
$$

The $S$-module of logarithmic differential 1-forms at $X[8]$ is defined by

$$
\begin{aligned}
\Omega^{1}(X) & =\left\{\omega \in \frac{1}{Q} \Omega_{\mathrm{alg}}^{1}(V): d \omega \in \frac{1}{Q} \Omega_{\mathrm{alg}}^{1}(V)\right\} \\
& =\left\{\frac{\omega}{Q}: \omega=\sum_{i=1}^{n} q^{i} d x_{i}, q^{i} \in S, l_{H} \mid d l_{H} \wedge \omega \text { for all } H \in X\right\} .
\end{aligned}
$$

$\Omega^{1}(X)$ is a $\mathbf{Z}$-graded module with (cf. $[24, \S 3]$ )

$$
\Omega^{1}(X)=\bigoplus_{k \geq-m} \Omega^{1, k}(X)
$$

where

$$
\Omega^{1, k}(X)=\Omega^{1}(X) \cap \frac{1}{Q} \Omega_{\mathrm{alg}}^{1, k+m}(V)
$$

is the k-vector space of homogeneous logarithmic 1 -forms at $X$ of degree $k$.

Dually (cf. Theorem 1.5(ii)), the $S$-module of logarithmic vector fields at $X$ is

$$
\operatorname{Der}(X)=\left\{\theta=\sum_{j=1}^{n} p_{j} \frac{\partial}{\partial x_{j}}: p_{i} \in S, l_{H} \mid \theta\left(l_{H}\right) \text { for all } H \in X\right\} .
$$

Definition 1.3 [15].The arrangement $X$ is free if $\Omega^{1}(X)$ is a free $S$-module.

From Theorem 1.5(ii) below we can see that, equivalently, $X$ is free iff $\operatorname{Der}(X)$ is a free $S$-module.

The most elementary formulation of freeness is due to Stanley [10]. He noted that $\operatorname{Der}(X)$ is isomorphic to the $S$-module $\left\{\mathbf{p} \in S^{n}: \mathbf{p}(H) \subseteq H\right.$ for all $H \in$ $X\}$ : the coefficient vectors of vector fields $\theta=\sum_{i=1}^{n} p_{i} \partial / \partial x_{i} \in \operatorname{Der}(X)$ can be interpreted as the polynomial functions $\mathbf{p}=\left(p_{1}, \ldots, p_{n}\right): \mathbf{k}^{n} \rightarrow \mathbf{k}^{n}$ which map every hyperplane of $X$ into itself.

This module is free if and only if it contains elements $\mathbf{p}_{1}=\left(p_{11}, \ldots, p_{1 n}\right)$, $\ldots, \mathbf{p}_{n}=\left(p_{n 1}, \ldots, p_{n n}\right)$ with $\operatorname{det}\left(p_{i j}\right)=Q$ (see Theorem 1.5(iii) below).

The question whether freeness of an arrangement is a combinatorial property $[15$, p. $293 ; 16$, p. $565 ; 10 ; 13$, p. 167] has become known as Terao's Conjecture. 
We will see in Example 4.1 that at least some restrictions are necessary for the validity of this conjecture. Thus a more specific formulation of Terao's Conjecture will be needed.

Definition 1.4. Terao's Conjecture holds ("TC holds") for a class of arrangements if for arrangements in this class, freeness is a combinatorial property.

In particular, Terao's Conjecture holds for a matroid $M$ if the freeness of the arrangements corresponding to $M$ does not depend on the specific representation of $M$. It holds for a class $\mathscr{M}$ of matroids if it holds for the class of arrangements whose abstract matroid is in $\mathscr{M}$.

Terao's Conjecture holds over $\mathbf{k}$ if it holds for the arrangements over $\mathbf{k}$.

In view of Example 4.1 below the strongest plausible conjecture seems to be that Terao's Conjecture holds over every field, or equivalently, in every characteristic.

Some special cases will be treated in the following section.

Now we review some algebraic facts about the modules $\Omega^{1}(X)$ of logarithmic forms at arrangements $X$ and their bases.

Theorem $1.5[8,15,17,18]$. (i) $\Omega^{1}(X)$ is a module of rank $n$. Thus the size of a minimal set of generators is given by $\operatorname{dim}_{\mathbf{k}} \mathbf{k} \otimes_{S} \Omega^{1}(X) \geq n$, with equality if and only if $\Omega^{1}(X)$ is free.

Hence the arrangement $X_{\mathbf{K}}$ over $\mathbf{K}$ obtained from $X$ by extension of the ground field is free if and only if $X$ is free, since $\Omega^{1}\left(X_{\mathbf{K}}\right)=\mathbf{K}\left[x_{1}, \ldots, x_{n}\right] \otimes_{S}$ $\Omega^{1}(X)$.

(ii) There is a nondegenerate pairing

$$
\operatorname{Der}(X) \times \Omega^{1}(X) \rightarrow S
$$

sending $(\theta, \omega)$ to $\omega(\theta)$; thus $\operatorname{Der}(X)$ and $\Omega^{1}(X)$ are reflexive $S$-modules, dual to each other. In particular, $\Omega^{1}(X)$ is free module if and only if $\operatorname{Der}(X)$ is free. Dual bases $\left\{\theta_{1}, \ldots, \theta_{n}\right\}$ with $\theta_{i}=\sum_{j} p_{i j} \partial / \partial x_{j}$ and $\left\{\omega_{1}, \ldots, \omega_{n}\right\}$ with $\omega_{i}=\sum_{j} q^{i j} d x_{j}$ are characterized by the conditions $\omega_{i}\left(\theta_{j}\right)=\delta_{i j}$, that is, the coefficient matrices satisfy $\left(q^{i j}\right)^{-1}=\left(p_{j i}\right)$. Thus if $X$ is free and $r(X)=n$, then the elements of a homogeneous basis of $\Omega^{1}(X)$ all have negative degree.

(iii) $\left\{\omega_{1}, \ldots, \omega_{n}\right\} \subseteq \Omega^{1}(X)$ is a basis of $\Omega^{1}(X)$ if and only if $\omega_{1} \wedge \cdots \wedge \omega_{n}=$ $c \frac{1}{Q} d x_{1} \wedge \cdots \wedge d x_{n}$ for some $c \in \mathbf{k}-\{0\}$, that is, iff $\operatorname{det}\left(q^{i j}\right)=\frac{c}{Q}$ in the notation of (ii). Similarly, $\left\{\theta_{1}, \ldots, \theta_{n}\right\} \subseteq \operatorname{Der}(X)$ is a basis of $\operatorname{Der}(X)$ if and only if $\operatorname{det}\left(p_{i j}\right)=c^{\prime} Q$ for some scalar $c^{\prime} \neq 0$.

(iv) If $\Omega^{1}(X)$ is free, then it has a homogeneous basis $\left\{\omega_{1}, \ldots, \omega_{n}\right\}$, where the $\omega_{i}$ are homogeneous of degree $-e_{i}$ for $1 \leq i \leq n$. The nonnegative integers $e_{i}(1 \leq i \leq n)$ do not depend on the homogeneous basis chosen. They are called the exponents of $X$. If $X$ is free, then $\chi(t) \cdot t^{n-r}=\prod_{i=1}^{n}\left(t-e_{i}\right)$. (This is Terao's celebrated "Factorization Theorem" [17, 9].) 
There are two large classes of degree arrangements "occurring in nature" that are known to be free: Coxeter arrangements (and more generally arrangements corresponding to groups generated by reflections in good characteristic, see [8, $18])$ and supersolvable arrangements $[10,5]$. In fact, the following result holds:

Theorem 1.6 [24]. An arrangement is supersolvable if and only if in suitable coordinates it admits a "triangular" basis $\left\{\omega_{1}, \ldots, \omega_{n}\right\}$ with $\omega_{i}=\frac{1}{Q} \sum_{j \geq i} q^{i j} d x_{j}$.

In this case, by Theorem 1.5(iv), the exponents of $X$ coincide with the exponents of $L$ defined as the zeroes of the characteristic polynomial.

Note that, in particular, arrangements in dimension 2 are always supersolvable and thus free.

Furthermore some important constructions preserve freeness: localization at a subspace, forming sums of arrangements, and addition/deletion of hyperplanes under suitable numerical conditions.

Theorem 1.7 [15]. (i) Let $X$ be a free arrangement in $V$ and let $W$ be a subspace of $V$. Then the localization of $X$ at $W$, given by $X^{W}=\{H \in$ $X: W \subseteq H\}$, is again a free arrangement in $V$. The direct sum $X \oplus X^{\prime}$ of two arrangements over $\mathbf{k}$ is free if and only if both $X$ and $X^{\prime}$ are free.

(ii) Let $X$ be an arrangement in $V$ and let $H \notin X$ be a hyperplane. Then for $1 \leq i \leq n$ every two of the following statements imply the third:

$* X$ is free with exponents $e_{1}, \ldots, e_{i}, \ldots, e_{n}$.

$* X \cup\{H\}$ is free with exponents $e_{1}, \ldots, e_{i}+1, \ldots, e_{n}$.

$\left.* X\right|_{H}$ is free with exponents $e_{1}, \ldots, \hat{e}_{i}, \ldots, e_{n}$,

where $\left.X\right|_{H}=\{K \cap H: K \in X\}$ is the restriction of $X$ to $H$.

Furthermore, when $X$ and $X \cup\{H\}$ are both free, then for some $i$ the above three statements hold.

The proof of part (i) is simple commutative algebra. See [9] for the statement about direct sums. Observe that localization of an arrangement corresponds to a restriction of the underlying matroid to a flat. The question ("Orlik's Conjecture") whether freeness is always preserved under restriction to a hyperplane in the arrangement is still open, although some positive evidence exists.

Part (ii) of Theorem 1.7 is Terao's “Addition/Deletion Theorem." Its original proof required heavy commutative algebra tools. For simpler proof variants see $[24, \S 5]$.

The following proposition is a basic technical fact used in the following. Its special case $\left|X_{1}\right|=1$ is [24, Lemma 6.1], where it was a direct corollary to the "Strong Preparation Lemma" [24, Theorem 5.1].

The general case uses the notion of "line closure" from [4]. We define a subset $Y \subseteq X$ to be line closed if with $H_{1}, H_{2} \in Y$, the set $Y$ contains every hyperplane $H \in X$ that contains $H_{1} \cap H_{2}$. Equivalently, $Y \subseteq X$ is line closed if with two elements of a 3-circuit in the matroid $M(X), Y$ also contains the third element (and thus the whole line determined by them). 
Proposition 1.8. Let $X=X_{0} \bullet X_{1}$, where $X_{0}$ and $X_{1}$ are line closed in $X$. Then

$$
\Omega^{1}(X)=\Omega^{1}\left(X_{0}\right)+\Omega^{1}\left(X_{1}\right) .
$$

Proof. We use induction on $\left|X_{1}\right|$, where the case $X_{1}=\varnothing$ is trivial.

Let $\omega \in \Omega^{1}(X)$ and $H \in X_{1}$. Then $l_{H} \omega \in \Omega^{1}(X-\{H\})$ can by induction be written as $l_{H} \omega=\omega_{0}+\omega_{1}$ with $\omega_{0} \in \Omega^{1}\left(X_{0}\right)$ and $\omega_{1} \in \Omega^{1}\left(X_{1}-\{H\}\right)$.

Now $\omega$ is logarithmic at $H$, and thus $\omega_{0}+\left.\omega_{1}\right|_{H}=0$. But this implies

$$
\left.\omega_{0}\right|_{H}=-\left.\omega_{1}\right|_{H} \in \Omega^{1}\left(\left.X_{0}\right|_{H}\right) \cap \Omega^{1}\left(\left.X_{1}\right|_{H}\right)=\Omega_{\mathrm{alg}}^{1}(H),
$$

because $\left.\left.X_{0}\right|_{H} \cap X_{1}\right|_{H}=\varnothing$ : there is no 3-circuit $\left\{H_{0}, H_{1}, H\right\}$ in $X$ with $H_{0} \in X_{0}, H_{1} \in X_{1}-\{H\}$. Now choose $\omega^{\prime} \in \Omega_{\mathrm{alg}}^{1}(V)$ such that $\left.\omega^{\prime}\right|_{H}=\left.\omega_{0}\right|_{H}=$ $-\left.\omega_{1}\right|_{H}$. Then $\omega_{1}+\omega^{\prime} \in \Omega^{1}\left(X_{1}\right)$ with $\omega_{1}+\left.\omega^{\prime}\right|_{H}=0$ and thus

$$
\frac{1}{l_{H}}\left(\omega_{1}+\omega^{\prime}\right) \in \Omega^{1}\left(X_{1}\right) \text {. }
$$

Similarly, we get

$$
\frac{1}{l_{H}}\left(\omega_{0}-\omega^{\prime}\right) \in \Omega^{1}\left(X_{0}+\{H\}\right),
$$

and hence

$$
\Omega^{1}(X)=\Omega^{1}\left(X_{0} \cup\{H\}\right)+\Omega^{1}\left(X_{1}\right) .
$$

But $H$ is not contained in a 3-circuit of $X_{0} \cup\{H\}$, hence by [24, Lemma 6.1] we have

$$
\Omega^{1}\left(X_{0} \cup\{H\}\right)=\Omega^{1}\left(X_{0}\right)+\Omega^{1}(\{H\}),
$$

and from $\Omega^{1}(\{H\}) \subseteq \Omega^{1}\left(X_{1}\right)$ the claim follows.

Lemma 1.9. If $X$ is free and $H \in X$, then $\Omega^{1}(X)$ has a basis that contains $d l_{H} / l_{H}$.

Proof. We choose coordinates such that $l_{H}=x_{1}$. Let $\theta_{1}, \ldots, \theta_{n}$ be a basis for the dual module $\Omega^{1}(X)^{*}=\operatorname{Der}(X)$ with $\theta_{i}=\sum_{j=1}^{n} p_{i j} \partial / \partial x_{j}$ (for $1 \leq i \leq$ $\left.n, p_{i j} \in S\right)$. Then by [15, p. 296] we may assume that $\theta_{1}$ is the Euler vector field $\theta_{1}=\sum_{j=1}^{n} x_{j} \partial / \partial x_{j}$. Now $\theta_{i} \in \operatorname{Der}(X)$ implies $l_{H} \mid \theta_{i}\left(l_{H}\right)$, that is, $x_{1} \mid p_{i 1}$ for $1 \leq i \leq n$. Thus, putting $\theta_{1}^{\prime}:=\theta_{1}$ and $\theta_{i}^{\prime}:=\theta_{i}-p_{i 1} \theta_{1} / x_{1}$ for $1<i \leq n$, we get a basis $\left\{\theta_{i}^{\prime}=\sum_{j=1}^{n} p_{i j}^{\prime} \partial / \partial x_{j}: 1 \leq i \leq n\right\}$ with $p_{i 1}^{\prime}=0$ for $i>1$.

The dual basis $\left\{\omega_{i}^{\prime}: 1 \leq i \leq n\right\}$ then contains $\omega_{1}^{\prime}=d x_{1} / x_{1}=d l_{H} / l_{H}$. (Essentially the same argument was used before in [25].)

\section{Projective uniqueness}

For large classes of matroids, Terao's Conjecture over a fixed field turns out to be true for the geometric reason that they are (essentially) projective unique. In this section, we will treat three main cases of this: binary matroids (represented over arbitrary fields), matroids over $\mathrm{GF}(3)$, and matroids over $\mathrm{GF}(4)$. 
Definition 2.1. (i) A representation of a simple matroid of rank $r$ is an order preserving injection $\phi: L \rightarrow \mathrm{PG}(r-1, \mathbf{k})$ of (the lattice of flats of) the matroid $L$ to the subspace lattice of $\mathbf{k}^{r}$.

(ii) A matroid $L$ is projectively unique over $\mathbf{k}$ if every two such representations of $L$ over $\mathbf{k}$ coincide up to an automorphism of $\mathrm{PG}(r-1, \mathbf{k})$, that is, for any two representations $\phi_{1}, \phi_{2}: L \rightarrow \mathrm{PG}(r-1, \mathbf{k})$ there is an automorphism $\rho$ of $\mathrm{PG}(r-1, \mathbf{k})$ such that $\rho \circ \phi_{1}=\phi_{2}$.

Observe that the condition in (ii) is vacuous if the matroid considered is not representable over $\mathbf{k}$ : for ease of notation we will always consider a matroid that is not representable over a given field as projectively unique over that field.

To proceed, we need some information about the automorphisms of $\mathrm{PG}(r-1, \mathbf{k})$. This is provided by the following corollary to the "(First) Fundamental Theorem of Projective Geometry".

Lemma 2.2 [1, p. 88; 2, p. 44].The automorphism group of $\mathrm{PG}(r-1, \mathbf{k})$ is generated by the linear automorphism group $\operatorname{PGL}(r-1, \mathbf{k}$ ) (induced from the canonical action of the general linear group on $\mathbf{k}^{r}$ ) together with the automorphisms induced by field automorphisms of $\mathbf{k}$.

From this we get the following statement.

Proposition 2.3. If $L$ is projectively unique over $\mathbf{k}$, then Terao's Conjecture holds for $L$ over $\mathbf{k}$.

Proof. Automorphisms of $\mathrm{PG}(r-1, \mathbf{k}$ ) (as described in Lemma 2.2) induce semilinear automorphisms of $\Omega^{1}(X)$ and do not affect freeness of this module over $S$.

Definition 2.1(ii) of projective uniqueness follows Kahn [4], allowing semilinear transformations induced by field automorphisms. This is weaker than the definition by Brylawski and Lucas [3] and White [21] which allows only linear transformations. In fact Kahn's main result (Theorem 2.6 below) is false for this stronger definition: over a field of characteristic 2 like GF(4) only binary matroids are unique in the Brylawski-Lucas sense. Thus Kahn's definition is more suitable when working over fields with nontrivial automorphisms. However, with it the statement of Proposition 2.4(i) is slightly weaker than what White (in his 1971 thesis, see [21]) and Brylawski and Lucas [3] actually proved.

Proposition 2.4 [21, 3]. (i) Binary matroids are projectively unique over every field.

(ii) Matroid representations over $\mathrm{GF}(3)$ are projectively unique.

With Proposition 2.3 these by now classical statements about projective uniqueness translate into proofs of Terao's Conjecture for special classes of matroids.

Theorem 2.5. (i) Terao's Conjecture holds for binary arrangements over fields of fuxed characteristic.

(ii) Terao's Conjecture holds over $\mathbf{k}=\mathrm{GF}(3)$. 
For part (i) we use the facts that freeness is stable under field extension (Theorem 1.5(i)) and that any two fields of the same characteristic have isomorphic extension fields (this is a well-known algebraic fact: the tensor product of the two fields over the prime field modulo any maximal ideal will do).

Now, as the four point line shows, the representation of matroids is not unique over any field other than $\mathrm{GF}(2)$ and $\mathrm{GF}(3)$.

However, over GF(4) enough uniqueness survives. In fact, we can use the following highly nontrivial result recently obtained by Kahn.

Theorem 2.6 [7]. 3-connected matroids are projectively unique over $\mathrm{GF}(4)$.

As before, this immediately implies Terao's Conjecture for 3-connected matroids over $\mathrm{GF}(4)$. However, we can prove more.

Theorem 2.7. Terao's Conjecture holds over $\mathrm{GF}(4)$.

Proof. We can assume that $r(X)=n$ and that $X$ is not a direct sum of arrangements (that is, the matroid $M$ is connected), because a direct sum of two arrangements is free if and only if both summands are free, by Theorem 1.7(i).

If $L$ is 3-connected, then we are done by Theorem 2.6. Otherwise, we get a 2-separation $X=X_{0} \bullet X_{1}$, with $r\left(\bar{X}_{0} \cap \bar{X}_{1}\right) \leq 1$.

Case 1. If $r\left(\bar{X}_{0} \cap \bar{X}_{1}\right)=0$, then $\bar{X}_{0} \cap \bar{X}_{1}=\varnothing$, and we get from Proposition 1.8 that

$$
\Omega^{1}(X)=\Omega^{1}\left(X_{0}\right)+\Omega^{1}\left(X_{1}\right) .
$$

Now assume that $X$ was free. Then for $i=0,1$ the module $\Omega^{1}\left(X_{i}\right)$ is free, thus the negative degree part of $\mathbf{k} \otimes \Omega^{1}\left(X_{i}\right)$ has dimension $r\left(X_{i}\right)$. With $\Omega^{1}\left(X_{0}\right) \cap \Omega^{1}\left(X_{1}\right)=\Omega_{\mathrm{alg}}^{1}(V)$ this implies

$$
\operatorname{dim} \mathbf{k} \otimes \Omega^{1}(X) \geq r\left(X_{0}\right)+r\left(X_{1}\right)>r(X)=n .
$$

Hence $\Omega^{1}(X)$ can never be free, and thus TC holds for $L$.

Case 2. If $r\left(\bar{X}_{0} \cap \bar{X}_{1}\right)=1$, we can assume $\bar{X}_{0} \cap \bar{X}_{1}=\{H\}$ with $H \in X_{1}$, and put $X_{2}:=X_{0} \cup\{H\}$. Now TC holds for $X_{1}$ and $X_{2}$ by induction (on the rank). Now if $X$ is free, then so are $X_{1}$ and $X_{2}$ : they are localizations of $X$ (Theorem 1.7(i)). Conversely, if $X_{1}$ and $X_{2}$ are free, we may by Lemma 1.9 choose homogeneous bases $\mathscr{B}_{i}$ for $\Omega^{1}\left(X_{i}\right)$ with $d l_{H} / l_{H} \in \mathscr{B}_{i}$, for $i=1,2$. Then the elements of negative degree in $\mathscr{B}_{1} \cup \mathscr{B}_{2}$ form a basis for $\Omega^{1}(X)$ (Basis Criterion of Theorem 1.5(iii)). With this we know that $X$ is free if $X_{1}$ and $X_{2}$ are both free, and thus TC holds for $X$.

The argument in the last proof is in fact more general. It shows that for every class of matroids that is closed under deletions (in particular, for arrangements over a fixed field), it is sufficient to prove Terao's Conjecture for 3-connected matroids, or, equivalently, that every minimal counterexample to Terao's Conjecture is 3-connected. 
The observations by Kahn [7] about representations over larger fields also apply to our problem: projective uniqueness breaks down over $\operatorname{GF}(q)$ for $q \geq$ 5 , and sufficiently high connectivity of the matroid is not sufficient to guarantee it.

In fact, although free arrangements seem to be "rigid" in some vague sense, they are by no means projectively unique in general. For example, for supersolvable arrangements arbitrary generic points on the modular line can be added without destroying supersolvability.

We believe that Terao's conjecture over larger fields is in fact false, and it should be possible to construct counterexamples that have a finite representation space. If the construction of representations for such a matroid uses the existence of zeroes of a quadratic polynomial, and if the zeroes of this polynomial lie in the prime field of $\mathbf{k}$, then it is conceivable that one of the representations is free by supersolvable resolution, whereas the other is not free. The same could as well happen over $\mathbf{Q}, \mathbf{R}$, or $\mathbf{C}$.

\section{Supersolvable RESOlutions}

Definition 3.1 [15, p. 308]. An arrangement is inductively free if there is a sequence

$$
\varnothing=X_{0} \subset X_{1} \subset \cdots \subset X_{m}=X
$$

of subarrangements such that $X_{i}$ is free and $\left|X_{i}\right|=i$ for all $0 \leq i \leq m$. Being inductively free is a combinatorial property for arrangements of dimension $n \leq 3$ : this is clear from Terao's Addition/Deletion Theorem 1.7(ii) and the fact that exponents are combinatorial (Theorem 1.5(iv)). If Orlik's Conjecture (stating that restrictions of free arrangements to their hyperplanes are free) is true, then the restriction $n \leq 3$ can be dropped.

Definition 3.2. Let $X$ be an arrangement in $V$. A supersolvable resolution of $X$ is a finite sequence

$$
X=Y_{0} \subset Y_{1} \subset \cdots \subset y_{k}=y
$$

such that $\left|Y_{i}\right|=|X|+i$ for $0 \leq i \leq k$, and $Y$ is supersolvable.

Trivially every supersolvable arrangement has a supersolvable resolution. On the other hand, the lattice of all subspaces of a finite dimensional vector space $V$ is modular and hence supersolvable; however, the set of all (central) hyperplanes in $V$ is not finite unless $V$ itself is finite. This motivates the following lemma:

Lemma 3.3. Every arrangement has a supersolvable resolution.

Proof. Let $X$ be an arrangement with $r(X)=n$. It is sufficient to show that $X$ is contained in a finite supersolvable arrangement $Y$. Given $X$, choose an arbitrary line (1-dimensional subspace) $l$ in $V$. Now let $X^{\prime}$ be the arrangement in $V$ obtained from $X$ by adding to it all the hyperplanes in $V$ which contain both $l$ and the intersection of two hyperplanes of $X$ that do not contain $l$, that is,

$$
X^{\prime}=X \cup\left\{\operatorname{span}\left\langle l, H^{\prime} \cap H^{\prime \prime}\right\rangle: H^{\prime} \neq H^{\prime \prime} ; l \nsubseteq H^{\prime} \cap H^{\prime \prime} ; H^{\prime}, H^{\prime \prime} \in X\right\} .
$$


In $X^{\prime}, l$ is a modular line. Now, by induction on the rank, we can add a finite number of planes containing $l$ to $X^{\prime}$, such that in the resulting arrangement $Y$, the localization $Y / l$ is supersolvable. But with $Y / l$ supersolvable and $l$ a modular coatom of $L(Y), Y$ is supersolvable.

This proof with suitable choice of $l$ sometimes produces small supersolvable resolutions.

Definition 3.4. An arrangement $X$ in $V$ is called free by supersolvable resolution if it has a supersolvable resolution consisting of free arrangements.

With this, we get the following technique to check freeness that works especially well for arrangements of rank 3 . Let $X$ be an arrangement with characteristic polynomial $\prod_{i=1}^{r}\left(t-e_{i}\right)$. If $X$ is free, then these $e_{i}$ are the exponents of $X$. Now we attempt to find a supersolvable resolution $X=$ $Y_{0} \subset \cdots \subset Y_{k}=Y$ such that for every $i$, the exponents of the restriction $\left.Y_{i-1}\right|_{H_{i}}\left(Y_{i}=Y_{i-1} \cup\left\{H_{i}\right\}, 1 \leq i \leq k\right)$ form a submultiset of the exponents of $Y_{i-1}$, that is $\chi\left(\left.Y_{i-1}\right|_{H_{i}}\right) \mid \chi\left(Y_{i-1}\right)$. This is easy to check when $\left.Y_{i-1}\right|_{H_{i}}$ is 2-dimensional. By Addition Theorem 1.7(ii), we are then done. Conversely, if $X \subseteq Y, Y$ is free (e.g., supersolvable) and $\chi_{X}(t)$ factors over $\mathbf{Z}$, then we know that $e_{i}(X) \leq e_{i}(Y)$, in some ordering of the exponents, by the argument used to prove [24, Proposition 5.2]. This for some arrangements $X$ yields a simple proof that $X$ is not free. We demonstrate this technique by a sequence of examples.

Example 3.5 $[6,5]$. The arrangements $D_{n}^{k}$ defined over a field $\mathbf{k}$ with $\operatorname{char}(\mathbf{k})$ $\neq 2$ by

$$
Q_{n}^{k}=\prod_{i=1}^{k} x_{i} \prod_{1 \leq i<j \leq n}\left(x_{i}^{2}-x_{j}^{2}\right)
$$

are free by supersolvable resolution with exponents $[1,3, \ldots, 2 n-3, n+k-$ 1]. For this we note that $D_{n}^{n}$ is supersolvable with exponents $[1,3, \ldots, 2 n-$ $3,2 n-1]$. This is the Coxeter arrangement $B_{n}$. For arbitrary $k$, we hence get a supersolvable resolution $D_{n}^{0} \subset D_{n}^{1} \subset \cdots \subset D_{n}^{n}$. We observe that $\left.D_{n}^{k}\right|_{\left\{x_{k}=0\right\}} \simeq$ $D_{n-1}^{n-1}$ has exponents $[1,3, \ldots, 2 n-3]$ for all $k$ by induction on $n$, thus we are done. [5] proved the slightly stronger fact that the arrangements $D_{n}^{k}$ are actually inductively free, but our argument for freeness is simpler. $D_{n}^{k}$ is not supersolvable in general, as can be seen from the Coxeter arrangements $D_{n}=D_{n}^{0}$ for $n \geq 4$ and from $D_{3}^{1}$ : this arrangement corresponds to the nonFano matroid. These examples show that over every field with characteristic different from 2 there are free arrangements that are not supersolvable.

The following is an example of an arrangement that is free by supersolvable resolution, but not inductively free: 
Example 3.6. Let $T$ be the arrangement in $\mathbf{R}^{3}$ sketched by [15, p. 311]. We define it by the following matrix

$$
\left(\begin{array}{ccc}
-1 & 0 & 1 \\
1 & 0 & 1 \\
0 & -1 & 1 \\
0 & 1 & 1 \\
0 & 0 & 1 \\
1 & 1 & 0 \\
-1 & 1 & 0 \\
1 & 0 & 0 \\
0 & 1 & 0 \\
t & t+1 & 1 \\
-t & t+1 & 1 \\
t & -(t+1) & 1 \\
-t & -(t+1) & 1 \\
t^{2} & 0 & 1 \\
-t^{2} & 0 & 1 \\
t & 1-t & 1 \\
t & t-1 & 1 \\
-t & 1-t & 1 \\
-t & t-1 & 1
\end{array}\right)
$$

where $t^{2}-2 t-1=0$ has to be satisfied. Note that with this $T$ is representable over $\mathbf{Q}[\sqrt{2}]$ (with $t_{1 / 2}=1 \pm \sqrt{2}$ ), but not over $\mathbf{Q}$. Figure 1 is an affine representation of the real arrangement obtained for $t=1+\sqrt{2}$, whereas the figure in $[15$, p. 311$]$ corresponds to $t=1-\sqrt{2}$. The characteristic polynomial for $T$ is computed as $\chi_{T}(t)=(t-1)(t-9)(t-9)$. However, every restriction $\left.T\right|_{H}$ is free with exponents [1,7]. Thus, $T$ is not inductively free. Now use the supersolvable resolution

$$
T=Y_{0} \subset Y_{1} \subset Y_{2} \subset \cdots \subset Y_{6}=Y
$$

given by $Y_{i}=Y_{0} \cup\left\{H_{i}\right\} \quad(1 \leq i \leq 6)$, where the $H_{i}$ are the hyperplanes given by

$$
\left(\begin{array}{ccc}
t & 0 & 1 \\
-t & 0 & 1 \\
1-2 t & 0 & 1 \\
2 t-1 & 0 & 1 \\
-t & 0 & t+2 \\
t & 0 & t+2
\end{array}\right)
$$

(They would correspond to extra "vertical" lines in Figure 1.) Now $Y$ is supersolvable (and thus free) with exponents 1, 13, 11, and in fact repeated application of the Addition/Deletion Theorem yields that $T$ is free by supersolvable resolution with exponents $1,9,9$. 


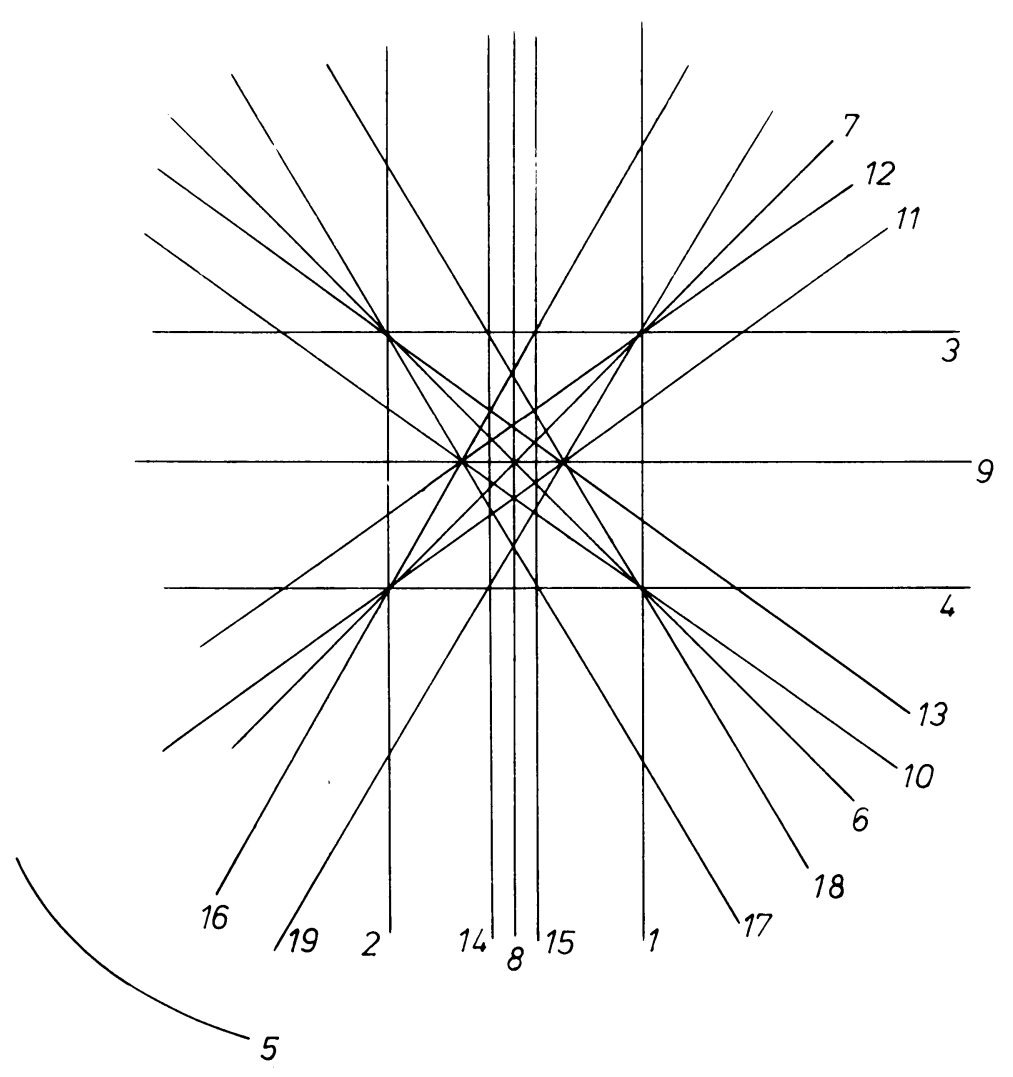

FIGURE 1

Note that $T$ is projectively unique over $\mathbf{Q}[\sqrt{2}]$, but not over $\mathbf{R}$ or $\mathbf{C}$. Since freeness is stable under field extension by Theorem 1.5(i), the matroid of $T$ cannot produce a counterexample to $\mathrm{TC}$ in characteristic 0 . It does not produce one in positive characteristic, either: over fields like $\mathrm{GF}(7), \mathrm{GF}(23), \mathrm{GF}(31)$, etc. that contain a root of $t^{2}-2 t-1$ and admit representations of $T$, the supersolvable resolution described above always exists, and $T$ is again free by supersolvable resolution.

Example 3.7. Let $X$ be an arrangement consisting of a pencil of five planes in $\mathbf{k}^{3}$ and two extra planes whose intersection is not contained in a plane of the pencil (cf. Figure 2). Then $X$ is not supersolvable, but its characteristic polynomial splits as $\chi(t)=(t-1)(y-3)^{3}$ [12, Example 4.7].

To see that $X$ cannot be free, we observe that $X$ has a supersolvable resolution of length 1 given by

$$
X \subset X \cup\{H\}=Y,
$$

where $H$ is the plane spanned by the axis of the pencil and the intersection line of the two extra planes. 


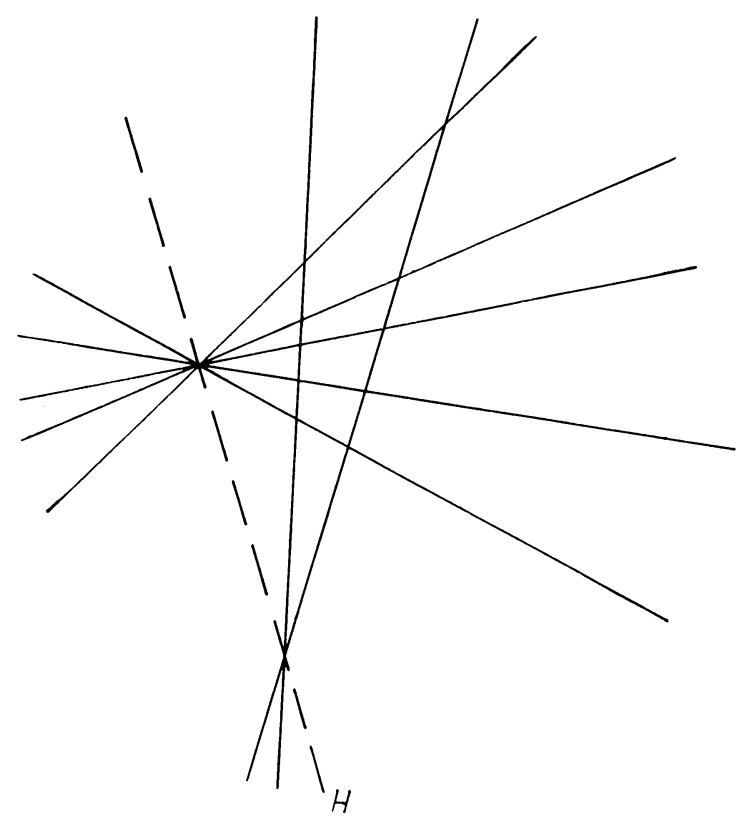

FIGURE 2

Now $Y$ is supersolvable (and thus free) with $\chi_{Y}(t)=(t-1)(t-5)(t-2)$, and thus $X$ cannot be free, by Theorem 1.7(ii).

\section{TWO EXAMPLES}

- The following extended example illustrates several phenomena that can occur when a matroid is represented over fields of different characteristics:

- The freeness of an arrangement can depend on the field characteristic.

- The existence of specified supersolvable resolutions can depend on the characteristic of the field, even if the matroid is projectively unique over every field considered.

- There are free, nonsupersolvable arrangements over fields of characteristic 2.

- There are free, not inductively free arrangements of nine respectively ten hyperplanes that are even representable in characteristic 2 . (The only previously published example is the arrangement of 19 planes in Example 3.6, which is not representable over small fields.)

Example 4.1. Let $M=\operatorname{AG}(2,3)$ be the matroid of the affine plane of order 3. This matroid (and the corresponding hyperplane arrangement) can be represented over a field $\mathbf{k}$ if and only if $\mathbf{k}$ contains a root of $\omega^{2}-\omega+1$. Every 
such $\omega$ determines a coordinatization by (compare $[14$, p. 19]):

$$
\left(\begin{array}{lll}
1 & 0 & 0 \\
0 & 1 & 0 \\
1 & \omega & 0 \\
0 & 0 & 1 \\
1 & 1 & 1 \\
\omega & \omega & 1 \\
\omega & 0 & 1 \\
1 & \omega & 1 \\
0 & 1 & 1
\end{array}\right) .
$$

Thus $M$ is coordinatizable in every characteristic; in particular it is representable over $\operatorname{GF}(3)$ (with $\omega=-1$ ), over $\mathrm{GF}(4)$, and over larger prime fields like $\operatorname{GF}(13)$ (with $\omega=4,-3$ ), $\mathrm{GF}(19)$ (with $\omega=8,-7$ ), etc.

Note that in these last two cases, there is no automorphism of $\mathbf{k}$ that carries $\omega$ to $\omega^{-1}$, and thus the representations of $M$ over such fields of GF(13) and $\mathrm{GF}(19)$ are not projectively unique. (However, this changes if automorphisms of the matroid are admitted in the definition of projective uniqueness.) $M$ is also realizable over $Q[\sqrt{-3}]$ and thus over $\mathbf{C}$, but it is not realizable over $\mathbf{R}$ or any subfield of $\mathbf{R}$ : in fact, it would violate the Sylvester-Gallai Theorem there. The characteristic polynomial of $M$ is $\chi(t)=(t-1)(t-4)^{2}$.

Case 1. If $\mathbf{k}$ is a field of characteristic 3 , then $M$ is representable over $\mathbf{k}$ and projectively unique. The corresponding hyperplane arrangement $X_{M}$ has a supersolvable resolution of length 1: in fact, adding an extra hyperplane given by the vector $A=(110)$, we get a supersolvable arrangement with exponents 1, 3, 6 (as sketched in Figure 3a). Thus it follows from the Addition/Deletion Theorem 1.7(ii) that $X_{M}$ cannot be free. For this construction to work, the characteristic of $\mathbf{k}$ has to be 3 , because otherwise the lines (123), (456), and (789) of the matroid cannot be extended by a common point.

Case 2. If $\operatorname{char}(\mathbf{k}) \neq 3$, then every arrangement $X_{M}$ obtained from a representation of $M$ over $\mathbf{k}$ has a supersolvable resolution of length 2: we first add in the point $A=\left(\begin{array}{lll}1 & 10\end{array}\right)$ (on the intersection of (123) and (456)) and then the point $B=(-\omega 10)$ (on the intersection of (123) and (789)), as in Figure 3b.

This new arrangement is supersolvable with exponents 1, 4, 6 and with the Addition/Deletion Theorem it is easily checked that $X_{M}$ is in fact free by supersolvable resolution, with exponents 1, 4, 4. The Addition/Deletion Theorem in fact implies that $X_{M}-\{H\}$ is not free for any $H \in X_{M}$. Thus in particular $X_{M}$ is not inductively free in the sense of Definition 3.1.

The standard example of a free, not supersolvable arrangement corresponds to the non-Fano plane $F^{-}$, which is denoted $A_{1}(7)$ in [15], $D_{3}^{1}$ in [5] (see Example 3.5), and $X_{0}$ in [23] and [24]. This arrangement of rank 3 is in fact inductively free $[15,5]$. 


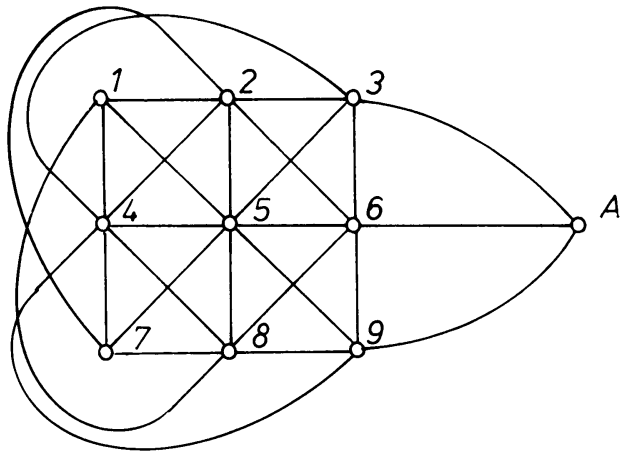

FIGURE 3a

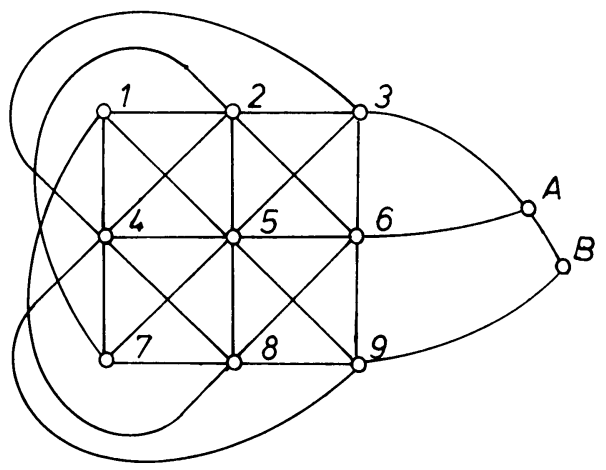

FIGURE 3b

However, it is well known that $F^{-}$is realizable over a field $\mathbf{k}$ if and only if $\operatorname{char}(\mathbf{k}) \neq 2$. It turns out that all published examples of free, not supersolvable arrangements are not binary. The matroid of Example 4.1 is representable in characteristic 2, but not over GF(2).

Our second example provides a free arrangement over $\mathrm{GF}(2)$ that is not supersolvable. It has rank 5 (as compared to the many examples of rank 3 over other fields) and considerable size. This is necessary, because binary free arrangements of rank at most four are always supersolvable: in the case $r=3$ this follows from [24, Corollary 7.6] together with the fact that 3-point lines in binary matroids are always modular, and in the case $r=4$ from a detailed analysis of the possible binary critical arrangements as defined in [24, §8]. Compare [26] for the combinatorial structure of supersolvable binary matroids.

Example 4.2. Let $M_{1}$ be the geometry over GF(2) of rank 5 obtained by deleting from PG $(4,2)$ the points (00001), (00011), (00101), (01001), (10001), (11001), and (10101). Since none of the deleted points is on the hyperplane $H=\left\{x_{5}=0\right\}$, we get that $M_{1}$ is supersolvable with exponents $1,2,4,8,9$. In fact $H$ is easily checked to be the only hyperplane from which no points have been deleted, and hence (in view of the exponents) the only modular hyperplane of $M_{1}$. The corresponding arrangement $X_{1}$ over $\mathrm{GF}(2)$ is supersolvable and thus free with exponents $1,2,4,8,9$. 
Now let $M_{2}$ be the geometry obtained from $M_{1}$ by deleting $x_{1}=(11110)$, and $X_{2}$ the corresponding arrangement. Let $H_{1} \in X_{1}-X_{2}$ be the hyperplane corresponding to $x_{1}$. Then since no two different points of $\operatorname{PG}(4,2)-M_{1}$ lie on the same line through $x_{1}$, we get that $\left.X_{2}\right|_{H_{1}}$ corresponds to the matroid $\operatorname{PG}(3,2)$ and is thus free with exponents $1,2,4,8$. Thus from Terao's Addition/Deletion Theorem 1.7(ii) we can conclude that $X_{2}$ is free with exponents $1,2,4,8,8$.

However, $X_{2}$ does not have a modular hyperplane, because in view of the exponents every modular hyperplane has to be complete (with exponents 1,2 , $4,8)$, and $M_{2}$ does not contain a complete hyperplane of $\mathrm{PG}(4,2)$.

\section{REFERENCES}

1. E. Artin, Geometric algebra, Interscience, New York, 1957.

2. R. Baer, Linear algebra and projective geometry, Academic Press, New York, 1952.

3. T. H. Brylawski and D. Lucas, Uniquely representable combinatorial geometries, Proc. 1973 Rome Internat. Colloq. Combinatorial Theory I, Accademia Nazionale dei Lincei, Rome, 1976, pp. 83-104.

4. M. D. Halsey, Line closed combinatorial geometries, Discrete Math. 65 (1987), 245-248.

5. M. Jambu and H. Terao, Free arrangements of hyperplanes and supersolvable lattices, Adv. in Math. 52 (1984), 248-258.

6. P. Orlik and L. Solomon, Coxeter arrangements, Singularities (P. Orlik, ed.), Proc. Sympos. Pure Math., vol. 40, Part 2, Amer. Math. Soc., Providence, R. I., 1983, pp. 269-291.

7. J. Kahn, On the uniqueness of matroid representations over $\mathrm{GF}(4)$, Bull. London Math. Soc. 20 (1988), 5-10.

8. K. Saito, Theory of logarithmic differential forms and logarithmic vector fields, J. Fac. Sci. Univ. Tokyo Sect. IA 27 (1980), 265-291.

9. L. Solomon and H. Terao, A formula for the characteristic polynomial of an arrangement, Adv. in Math. 64 (1987), 305-325.

10. R. P. Stanley, T-free arrangements of hyperplanes, Progress in Graph Theory (J. A. Bondy and U. S. R. Murty, eds.), Academic Press, New York, 1984, p. 539.

11. _ Modular elements of geometric lattices, Algebra Universalis 1 (1971), 214-217.

12. __ Supersolvable lattices, Algebra Universalis 2 (1972), 197-217.

13. __ Enumerative combinatorics, Volume 1, Wadsworth, Belmont, Calif., 1986.

14. B. Sturmfels, Computational algebraic geometry of projective configurations, Symbolic Computations in Geometry (B. Sturmfels and N. White, eds.), Univ. of Minnesota, IMA Preprint Series \# 398, 1988, pp. 17-37.

15. H. Terao, Arrangements of hyperplanes and their freeness. I, J. Fac. Sci. Univ. Tokyo Sect. IA 27(2) (1980), 293-312.

16. The exponents of a free hypersurface, Singularities (P. Orlik, ed.), Proc. Sympos. Pure Math., vol. 40, Dart 2, Amer. Math. Soc., Providence, R. I., 1983, pp. 561-566.

17. _ Generalized exponents of a free arrangement of hyperplanes and Shephard-ToddBrieskorn formula, Invent. Math. 63 (1981), 159-179.

18. _ Free arrangements of hyperplanes over an arbitrary field, Proc. Japan Acad. Ser. A 59 (1983), 301-303.

19. D. J. A. Welsh, Matroid theory, Academic Press, New York, 1976.

20. N. White, ed., Theory of matroids, Cambridge Univ. Press, Cambridge, 1986.

21. __ Combinatorial geometries, Cambridge Univ. Press, Cambridge, 1987. 
22. T. Zaslavsky, Facing up to arrangements: face count formulas for partitions of space by hyperplanes, Mem. Amer. Math. Soc., vol. 1, no. 514, 1975.

23. G. M. Ziegler, Algebraic combinatorics of hyperplane arrangements, Ph. D. Thesis, MIT, 1987.

24. Combinatorial construction of logarithmic differential forms, Adv. in Math. 76 (1989), 116-154.

25. _ Multiarrangements of hyperplanes and their freeness, Proc. Internat. Conf. Singularities (Iowa City, 1986), Contemp. Math., vol. 90, Amer. Math. Soc., Providence, R.I., 1989, pp. 345-359.

26. __ Supersolvable binary matroids and modular constructions, preprint, 1988.

InStitut für MAthematik, Universität AUgsburg, Universitätsstr. 8, 8900, AUgsburg, WEST GERMANY 